\title{
Identifying chemogenetic interactions from CRISPR screens with drugZ
}

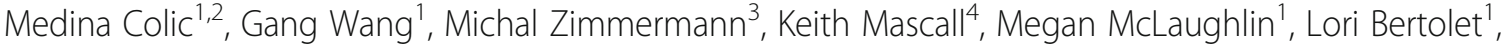 \\ W. Frank Lenoir ${ }^{1,2}$, Jason Moffat ${ }^{5,6}$, Stephane Angers ${ }^{4,7}$, Daniel Durocher ${ }^{3,6}$ and Traver Hart $^{*^{*}}$ (D)
}

\begin{abstract}
Background: Chemogenetic profiling enables the identification of gene mutations that enhance or suppress the activity of chemical compounds. This knowledge provides insights into drug mechanism of action, genetic vulnerabilities, and resistance mechanisms, all of which may help stratify patient populations and improve drug efficacy. CRISPR-based screening enables sensitive detection of drug-gene interactions directly in human cells, but until recently has primarily been used to screen only for resistance mechanisms.
\end{abstract}

Results: We present drugZ, an algorithm for identifying both synergistic and suppressor chemogenetic interactions from CRISPR screens. DrugZ identifies synthetic lethal interactions between PARP inhibitors and both known and novel members of the DNA damage repair pathway, confirms KEAP1 loss as a resistance factor for ERK inhibitors in oncogenic KRAS backgrounds, and defines the genetic context for temozolomide activity.

Conclusions: DrugZ is an open-source Python software for the analysis of genome-scale drug modifier screens. The software accurately identifies genetic perturbations that enhance or suppress drug activity. Interestingly, analysis of new and previously published data reveals tumor suppressor genes are drug-agnostic resistance genes in drug modifier screens. The software is available at github.com/hart-lab/drugz.

Keywords: CRISPR screens, Chemogenetic interactions, Drug resistance, Synthetic lethality

\section{Background}

The ability to systematically interrogate multiple genetic backgrounds with chemical perturbagens is known as chemogenetic profiling. While this approach has many applications in chemical biology, it is particularly relevant to cancer therapy, where clinical compounds or chemical probes are profiled to identify mutations that inform on genetic vulnerabilities, resistance mechanisms, or targets [1]. Systematic surveys of the fitness effects of environmental perturbagens across the yeast deletion collection [2] offered insight into gene function at a large scale, while profiling of drug sensitivity in heterozygous deletion strains identified genetic backgrounds that give rise to increased drug sensitivity [3]. Now, with the advent of CRISPR technology and its adaptation to pooled library screens in mammalian cells, high-resolution chemogenetic screens can be carried out directly in

\footnotetext{
* Correspondence: traver@hart-lab.org

${ }^{1}$ Department of Bioinformatics and Computational Biology, The University of

Texas MD Anderson Cancer Center, Houston, TX, USA

Full list of author information is available at the end of the article
}

human cells [4-7]. Major advantages to this approach include the ability to probe all human genes, not just orthologs of model organisms; the analysis of how druggene interactions vary across different tissue types, genetic backgrounds, and epigenetic states; and the identification of suppressor as well as synergistic interactions, which may preemptively indicate mechanisms of acquired resistance or pre-existing sources of resistant cells in heterogeneous tumor populations.

Design and analysis of CRISPR-mediated chemogenetic interaction screens in human cells can be problematic. Positive selection screens identifying genes conferring resistance to cellular perturbations typically have a high signal-to-noise ratio, as only mutants in resistance genes survive. This approach has been used to identify genes conferring resistance to targeted therapeutics, including BRAF and MEK inhibitors, as well as other drugs [5-13]. Conversely, negative selection CRISPR screens require growing perturbed cells over 10 or more doublings to allow sensitive detection of genes whose knockout leads to moderate fitness defects. 
Adding the detection of drug interactions to these experiments necessitates dosing at sub-lethal levels to balance between maintaining cell viability over a long time course and inducing drug-gene interactions beyond native drug effects [14-17].

In this study, we describe drugZ, an algorithm for the analysis of CRISPR-mediated chemogenetic interaction screens. We apply the algorithm to identify genes that drive normal cellular resistance to the PARP inhibitor olaparib in three cell lines. We demonstrate the greatly enhanced sensitivity of drugZ over contemporary algorithms $[7,18-20]$ by showing how it identifies more hits with higher enrichment for the expected DNA damage response pathway, and further how it identifies both synergistic and suppressor interactions. We further demonstrate the discovery of both synergistic and suppressor interactions in a single experiment with KRAS-mutant pancreatic cancer cell lines treated with an ERK inhibitor, and through reanalysis of published data. Interestingly, we observe a trend across several datasets where tumor suppressor genes score as drug suppressors, indicating a possible systematic source of false positives. We provide all software and data [21] necessary to replicate the analyses presented here; see "Availability of data and materials" below for links.

\section{Implementation}

\section{DrugZ algorithm}

We calculate the $\log _{2}$ fold change of each gRNA in the pool by normalizing the total read count of each sample (to $n=10$ million reads) at the same time point and taking the $\log$ ratio, for each replicate, of treated to control reads.

$$
\mathrm{fc}_{r}=\log _{2}\left[\frac{\operatorname{norm}\left(T_{t, r}\right)+\text { pseudocount }}{\operatorname{norm}\left(C_{t, r}\right)+\text { pseudocount }}\right]
$$

where:

- $\mathrm{fc}=$ fold change

- $\mathrm{r}=$ replicate indication

- $\mathrm{T}=$ treated sample

- $C=$ control sample

- $t$ = time point

- pseudocount $=$ default value is 5

We estimate the variance of each fold change by calculating the standard deviation of fold changes with similar abundance in the control sample:

$\operatorname{sort}\left(\mathrm{fc}_{r}\right)$ according $C_{r}($ descending $=$ True $)$

$$
\text { eb_std }_{\mathrm{fc}_{r}}=\sqrt{\frac{1}{N} \sum_{i}^{N}\left(f c_{r, i}-\mu\right)^{2}}
$$

where:

- $\quad$ eb_std $\mathrm{fc}_{\mathrm{f}_{r}}=$ estimated variance

- $N=$ number of fold changes with similar abundance (default $=1000)$

- $i=$ guide

- $\mathrm{fc}_{r, i}=$ fold change for each guide in a replicate

- $\mu=0$

and then calculate a $Z$-score for each fold change using this estimate:

$$
Z_{\mathrm{fc}_{r, i}}=\frac{\mathrm{fc}_{r, i}}{\text { eb_std }_{\mathrm{fc}_{r, i}}}
$$

The guide $Z$-score of all gRNA across all replicates is summed to get a gene-level sumZ score, which is then normalized (by dividing by the square root of the number of summed terms) to the final normZ (Fig. 1b):

$$
\text { norm } Z_{\text {gene } A}=\frac{\sum Z_{\mathrm{fc}_{r, i_{\text {geneA }}}}}{\sqrt{n}}
$$

A $P$ value is calculated from the normZ and corrected for multiple hypothesis testing using the method of Benjamini and Hochberg [22]. The open-source Python software can be downloaded from github.com/hart-lab/ drugz.

\section{DrugGS algorithm}

After empirical Bayes variance estimation approach is applied on normalized log-fold changes to calculate a $Z$ score for each guide, we applied Gibbs sampling to generate posterior distribution of fold changes for each gene.

$$
\begin{array}{cl}
\multicolumn{3}{c}{\text { Posterior } \sim \text { Likelihood } * \text { Prior }} \\
\mathrm{P}(\mu, \tau \mid \text { data })=\frac{P(\text { data } \mid \mu, \tau) * P(\mu, \tau)}{P(\text { data })} & \text { posterior } \\
P(\text { data } \mid \mu, \tau) & \text { likelihood } \\
P(\mu, \tau) & \text { prior }
\end{array}
$$

Each gene has a distribution composed of $Z$-scores for guides targeting that specific gene across replicates. Distribution is characterized as $\mathbb{N}(\mu, \tau)$, where $\tau$ is $\frac{1}{\sigma^{2}}$.

Both $\mu$ and $\tau$ have hyperparameters $\left(\mu: \mu, \sigma^{2}, \tau: a, b\right)$ that we initialize at the very start of sampling.

$P(\tau \mid$ data $) \sim \Gamma(\mathrm{a}, \mathrm{b})=$ Gamma prior with a (shape) and $\mathrm{b}$ (rate) hyperparameters 


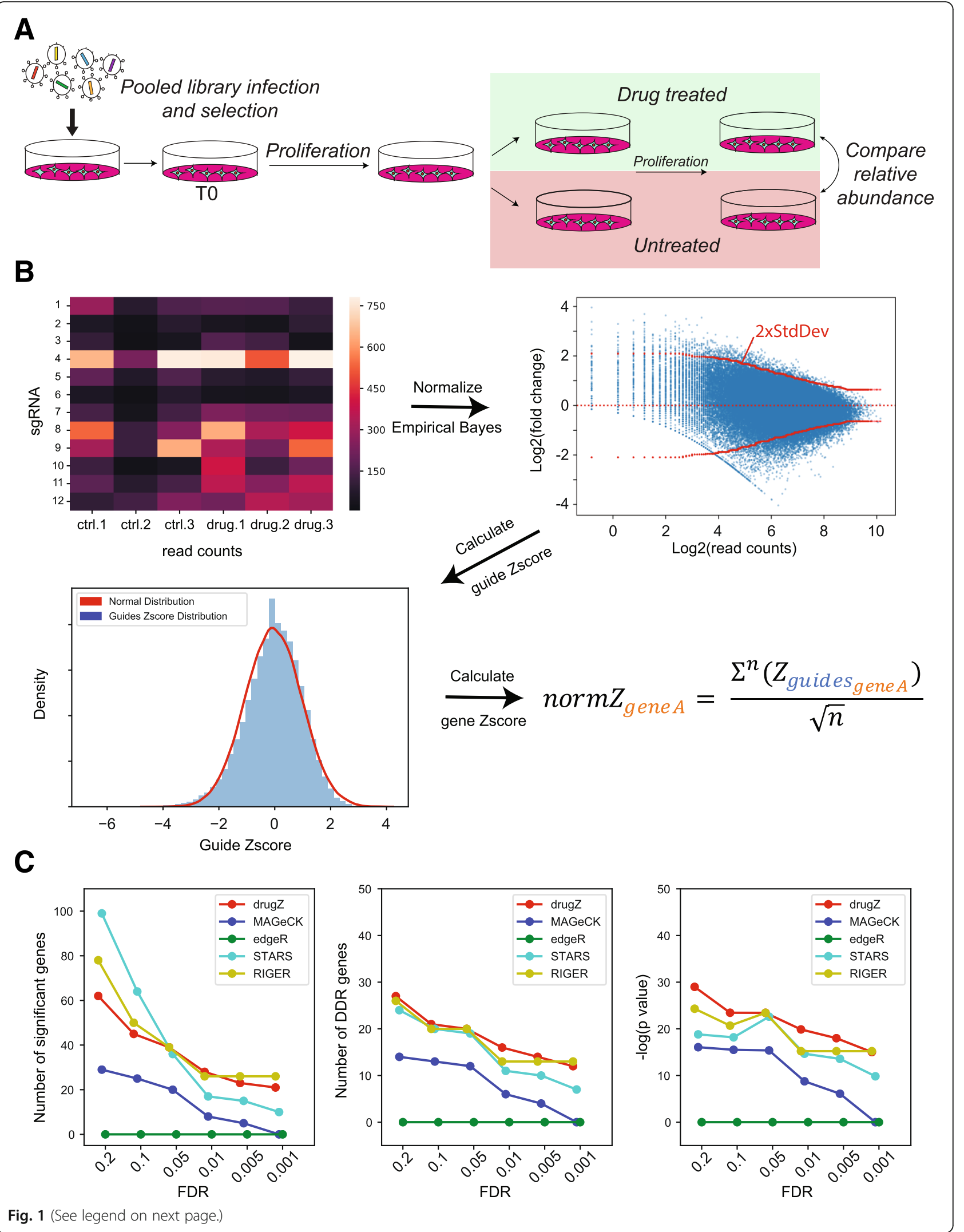


(See figure on previous page.)

Fig. 1 Workflow. a Experimental design. In a drug-gene interaction screen, cells are transduced with a pooled CRISPR library. Cells are split into drug-treated and untreated control samples, grown for several doublings; genomic DNA is collected; and the relative abundance of CRISPR gRNA sequences in the treated and control population is compared. $\mathbf{b}$ DrugZ processing steps include normalizing read counts, calculating fold change, estimating the standard deviation for each fold change, Z-score transformation, and combining guide scores into a gene score. c-e Comparing existing methods vs. drugZ for SUM149PT olaparib screen. DrugZ hits show strongest enrichments for DDR genes across a range of FDR thresholds. c Number of raw hits. $\mathbf{d}$ Number of annotated DNA damage response (DDR) genes in hits. e $-\log P$ values for DDR gene enrichment by hypergeometric test

$P(\mu \mid \tau$, data $) \sim \mathbb{N}\left(\mu, \sigma^{2}\right)=$ Normal prior with $\mu$ (mean) and $\sigma^{2}$ (variance) hyperparameters

We then update $\mu$ and $\tau$ with respect to their priors in every 1000 samples that we generate for each gene.

Equations to update $\mu$ :

$$
\begin{aligned}
\mu_{\text {update }} & =\frac{(n * \bar{y} * \tau)+\left(\mu_{\text {prior }} * \tau_{\text {prior }}\right)}{n * \tau+\tau_{\text {prior }}} \\
\sigma_{\text {update }} & =\frac{1}{\sqrt{n * \tau+\tau_{\text {prior }}}}
\end{aligned}
$$

Equations to update $\tau$ :

$$
\begin{aligned}
& a_{\text {update }}=a_{\text {prior }}+\frac{n}{2} \\
& b_{\text {update }}=b_{\text {prior }}+\sum\left(Z_{\left.\mathrm{fc}_{r, i}-\mu\right)^{2}}\right.
\end{aligned}
$$

where:

- $n=$ number of data points (guide $Z$-scores) for each gene

- $\bar{y}=$ actual mean of data points

From those 1000 newly sampled $\mu$ and $\tau$, we then calculate the mean and standard deviation. Each gene's $\mu$ posterior distribution's mean is what was converted into $Z$-score and used to compare with the drugZ normZ values.

$$
Z_{\text {geneA }}=\frac{\sum_{k=1}^{S} \mu_{k}}{S}
$$

where:

- $S=$ number of samples (in our case 1000)

- $k=$ sample

\section{Drug-gene interaction screens}

Olaparib screens were described in [14]. Temozolomide screens were described in [23].

\section{Cell culture}

hTERT RPE-1 (CRL-4000) and 293T (CRL-3216) cells were purchased from the ATCC and grown in Dulbecco's high glucose modified Eagle's medium (DMEM; HyClone) with $10 \%$ fetal bovine serum (FBS), $1 \times$ GlutaMAX (Gibco), $100 \mathrm{mM}$ sodium pyruvate (Gibco), 1× non-essential amino acids (NEAA), $1 \mathrm{X}$ penicillinstreptomycin (Pen/Strep), and $5 \mu \mathrm{g} \mathrm{ml}{ }^{-1}$ Plasmocure. Incubator conditions were kept at $37^{\circ} \mathrm{C}$ with $5 \% \mathrm{CO}_{2}$.

\section{Lentivirus production}

For production of the TKOV3 lentivirus, $9.0 \times 10^{6} 293 \mathrm{~T}$ cells were transfected with psPAX2 (lentiviral packaging; Addgene \#12260), pMD2.G (VSV-G envelope; Addgene \#12259), and TKOV3 (Toronto KnockOut CRISPR Library; Addgene \#90294) using X-tremeGENE 9 DNA transfection reagent (Sigma-Aldrich) in medium with lowered antibiotic concentration ( $0.1 \times$ Pen/Strep). The medium was replaced with viral harvest medium $($ DMEM $+1.1 \%$ BSA $+1 \times \quad$ Pen/Strep) $18 \mathrm{~h}$ posttransfection. Virus-containing supernatant was collected $\sim 24-48 \mathrm{~h}$ post-transfection, and fresh viral harvest medium was added to transfected plates. Viruscontaining supernatant was collected again $\sim 24$ later. The virus-containing supernatant was centrifuged to remove cell debris and stored at $-80^{\circ} \mathrm{C}$.

\section{CRISPR screening}

For transduction of the hTERT RPE-1 cells, the TKOv3 virus was added with $8 \mu \mathrm{g} / \mathrm{ml}$ Polybrene. For selection of the transduced cells, puromycin was introduced at a concentration of $20 \mu \mathrm{g} / \mathrm{ml}$ at $24 \mathrm{~h}$ post-infection (the hTERT cassette used to immortalize RPE1 cells contains a puromycin resistance marker, necessitating extreme puromycin concentrations for selection). Puromycin selection continued for $72 \mathrm{~h}$ post-transduction and completed upon the selection against the hTERT RPE-1 parental line as a control. Completion of selection was considered the initial time point $\left(T_{0}\right)$. The TKOv3transduced cells were split into technical replicates. To ensure proper coverage, $15 \times 10^{6}$ cells across $11 \times 15 \mathrm{~cm}$ dishes were used for infection with the TKOv3 virus per replicate. The chemotherapeutic drugs gemcitabine $(2$ $\mathrm{nM})$ and vincristine $(0.4 \mathrm{nM})$ were added to separate replicates, with one set of replicates receiving no drug 
treatment. Both drug-treated and untreated replicates were not allowed to reach confluence in the $15 \mathrm{~cm}$ dishes. Cells were lifted, counted, and re-plated at the coverage stated above, and the excess cell pellets were frozen at $-20^{\circ} \mathrm{C}$ as a time point. Once 8 doublings were reached from $T_{0}$, the screens were terminated and pellets frozen at $-20^{\circ} \mathrm{C}$. Coverage of screens was kept at 200 cells per gRNA.

The QIAamp Blood Maxi Kit (Qiagen) was used to isolate the genomic DNA (gDNA) from the frozen cell pellets. Guide sequences were enriched using PCR with HiFi HotStart ReadyMix (Kapa Biosystems) and primers targeting the guide region in the genomic DNA. A second round of PCR was performed with i5 and i7 primers to give each condition and replicate a unique multiplexing barcode. The final PCR products were purified using the E-Gel System (Invitrogen), normalized, and sequenced on the NextSeq500 system to determine the representation of guides under each treated and nontreated condition.

\section{Results and discussion}

We created the drugZ algorithm to fill a need for a method to identify chemogenetic interactions in CRISPR knockout screens. In a pooled library CRISPR screen, the relative starting abundance of each gRNA in the pool is usually sampled immediately after infection and selection. To identify genes whose knockout results in a fitness defect ("essential genes"), the cells are grown for several doublings and the relative abundance of gRNA is again sampled by deep sequencing of a PCR product amplified from genomic DNA template. The relative frequency of each gRNA is compared to starting gRNA abundance, and genes whose targeting gRNA show consistent dropout are considered essential genes.

In a chemogenetic interaction screen, the readout is different: the relative abundance of gRNA in a treated population is compared to the relative abundance of an untreated population at a matched time point (Fig. 1a). In this context, an experimental design with paired samples should be particularly powerful, as it removes a major source of variability across replicates.

To benchmark the method, we evaluated screens to identify modifiers of the response to the PARP inhibitor olaparib in three cell lines, RPE1-hTERT, HeLa, and SUM149PT [14]. The screens were performed using the TKOv1 library of 90k gRNA targeting 17,000 genes and are described in detail in [24]. After infection and selection, each cell line was split into 3 replicates, passaged at least once, and each replicate was further split into control and olaparib-treated populations (Fig. 1a).

The drugZ algorithm calculates a fold change for each gRNA in an experimental condition relative to an untreated control. A $Z$-score for each fold change is calculated using an empirical Bayes estimate of the standard deviation, by "borrowing" information from gRNA observed at a similar frequency (read count) in the control cells. Guide-level gene scores are combined into a normalized gene-level $Z$-scores called normZ, from which $P$ values are estimated from a normal distribution (Fig. 1b). We used drugZ to calculate normZ scores, $P$ values, and false discovery rates in SUM149PT breast cancer cells, which carry BRCA1 and TP53 mutations, +/- olaparib treatment [14]. We also analyzed the same data with four contemporary methods, STARS [7], MAGeCK [18], edgeR [19], and RIGER [20]. We noted that drugZ produced a moderate number of overall hits, relative to other methods, as FDR thresholds were relaxed (Fig. 1c). We evaluated the quality of the hits by measuring their functional coherence. The PARP inhibitor olaparib was developed specifically to exploit the observed synthetic lethal relationship between PARP1 and the BRCA1/BRCA2 genes [25, 26]. Subsequent studies have shown it to be effective against a general deficiency in homologous recombination repair, known as HRD [27]. We therefore calculated the enrichment of each hit set for genes in the DNA damage response (DDR) pathway as annotated in the Reactome database [28] and found that drugZ hits show strong enrichment for DDR genes across a range of FDR thresholds (Fig. $1 \mathrm{~d}, \mathrm{e})$, while the other methods show consistently lower enrichment. We observed similar trends in an olaparib screen in HeLa cells (Additional file 1: Figure S1A) but less overall effect in hTERT-immortalized RPE1 wildtype epithelial cells (Additional file 1: Figure S1B). The combination of larger sets of hits and greater enrichment for expected results indicates that drugZ accurately and sensitively identifies chemogenetic interactions.

The drugZ algorithm can also be used to identify suppressor interactions, that is, genes whose perturbation reduces drug efficacy. While BRCA1 mutation is synthetic lethal with PARP1, subsequent mutation of TP53BP1 is associated with acquired resistance to the PARP inhibitor [29]. Drug-gene interactions resulting in positive $Z$-scores reflect such suppressor interactions. Indeed, TP53BP1 is the 8th-ranked suppressor interaction in BRCA1-deficient SUM149PT cells, with a normZ score of 3.05. Similarly, newly described resistance gene C20orf196, now called SHLD1 [30-33], is the top-ranked suppressor.

Robustness to parameter choice and experimental design To evaluate the robustness of the drugZ approach, we conducted sensitivity analysis using data from the SUM149PT olaparib screen. The algorithm relies on two major tunable parameters, window size for empirical Bayes variance estimation and a monotone filter for the variance estimator (to ensure non-decreasing variance as 
read count decreases). The window size represents the number of neighboring gRNA, ranked by read count, to use to evaluate gRNA fold change variance. To evaluate the effect of varying window size, we ran the drugZ pipeline with window sizes in five increments from 100 to 1000; neither the number of hits, number of DDRannotated hits, nor enrichment $P$ value was affected by changing window size (Additional file 1: Figure S2a). We performed a similar analysis with and without enforcing the monotone filter and discovered marginally improved performance in the SUM149PT olaparib screen without enforcing monotonicity (Additional file 1: Figure S2b), but no such effect in Hela (T15) olaparib screen (Additional file 1: Figure S2c). We therefore left the filter in place.

We also tested the drugZ pipeline against a more statistically thorough, but computationally demanding, approach. After using the same empirical Bayes approach to calculate a $Z$-score for each guide, we applied Gibbs sampling to estimate the posterior distribution of fold changes for each gene (Additional file 1: Figure S3A). This method, which we termed drugGS, yielded results that are virtually identical to drugZ (Pearson correlation coefficient $=0.99$; Additional file 1: Figure $\mathrm{S} 3 \mathrm{~B}$ ) at $\sim 50 \times$ the computational cost (Additional file 1: Figure S3C). DrugGS is also available on github at https:// github.com/hart-lab/druggs.

\section{Experimental design considerations}

Highly effective CRISPR knockout screens are done with a variety of experimental designs, with varying numbers of replicates, degree of library coverage, determination of endpoint, and whether intermediate time points are included [5-7, 24, 34-40]. The olaparib drug-gene interaction screens described here were performed in triplicate in $15-\mathrm{cm}$ plates and passaged every 3 days, with drug added at day 6 and samples collected for sequencing at each passage starting at day 12 [14]. Using the optimized drugZ pipeline, we evaluated each time point in the SUM149PT screens. The screen's ability to resolve specific DNA damage response genes increased steadily from day 12 to day 18 (Fig. $2 \mathrm{a}-\mathrm{c}$ ), highlighting the importance of low-dose drug treatment (e.g., LD20). The extended timeframe for the experiment allows greater resolution of negative selection hits as they disappear from the population over several doublings.

Nevertheless, the screens are still quite noisy, necessitating several replicates for accurate assessment of druggene interactions. The experimental design of these screens involved control and drug-treated samples for each replicate, facilitating a paired-sample analysis across the three replicates (Additional file 1: Figure S4A). In contrast, an unpaired design (Additional file 1: Figure $\mathrm{S} 4 \mathrm{~A}$ ) requires comparing the means (or other aggregate metric) of the treated and untreated arms. In our experience, a paired-sample experimental design typically results in within-replicate samples clustering together (Additional file 1: Figure S4B), suggesting a paired-sample analysis would be more sensitive. Paired-sample analysis of three replicates in the olaparib screen clearly outperforms one- or two-replicate designs (Fig. 2b). Surprisingly, however, the paired-sample approach does not appear to offer significant benefits over an unpaired approach: when taking the mean fold change across experimental samples and comparing it to the mean fold change across control samples (Additional file 1: Figure S4A), the results are nearly identical to analysis of three paired samples (Fig. $2 \mathrm{~d}-\mathrm{f}$ ). Indeed, treating samples as paired or unpaired produced highly correlated results (rho $>=0.96$ ) in all three olaparib screens (Additional file 1: Figure S4c-e), and the functional enrichment analysis in SUM149PT cells showed virtually no difference when performing pairedsample or unpaired-sample analysis (Additional file 1: Figure S4f-h).

\section{A general-use algorithm for drug-gene interactions}

To ensure that the drugZ algorithm is not overspecialized for the strong chemogenetic profile of PARP inhibitors, we applied it to a separate set of drug interaction screens in pancreatic cancer cell lines using the ERK1/2 inhibitor SCH772984. Oncogenic mutations in KRAS drive constitutive signaling in the MAP kinase pathway and are associated with proliferation and survival signals. Consistent with current models of $R A S$ pathway activation, knockout of inhibitor target MAPK1 has strong synthetic sick/lethal or negative interactions with ERK inhibitor in two of the cell lines, MiaPaca and YAPC $($ FDR $<0.1$; Fig. 3a-d). In the third cell line, HPAF-II, the top synthetic interactors were drug transporter $A B C G 2$ and MAPK3. Activity of this drug resistance gene may account for this cell line's resistance to ERK inhibition and the lack of other synthetic effectors in this screen. Drug transporter $A B C C 4$ is synthetic lethal in MiaPaca cells, indicating multiple routes of drug resistance for this molecule. Ubiquitin ligase adapter KEAP1 is among the top suppressors of ERK inhibitor activity in three cell lines (Fig. 3a-d). KEAP1 loss of function was identified as a modulator of MAP kinase pathway inhibitors in a panel of positive selection screens in multiple cell lines [11], suggesting a context-dependent model for predicting ERK inhibitor activity (Fig. 3e). Notably, the ERK inhibitor screens yielded a small number of discrete synthetic and suppressor hits, in contrast with the PARP inhibitor screens, which showed broad interaction across the HR pathway, confirming the general applicability of drugZ in detecting drug-gene interactions.

We additionally reanalyzed data from a set of temozolomide (TMZ) drug modifier screens in patient-derived glioblastoma cell lines [23]. The screens clearly indicated 


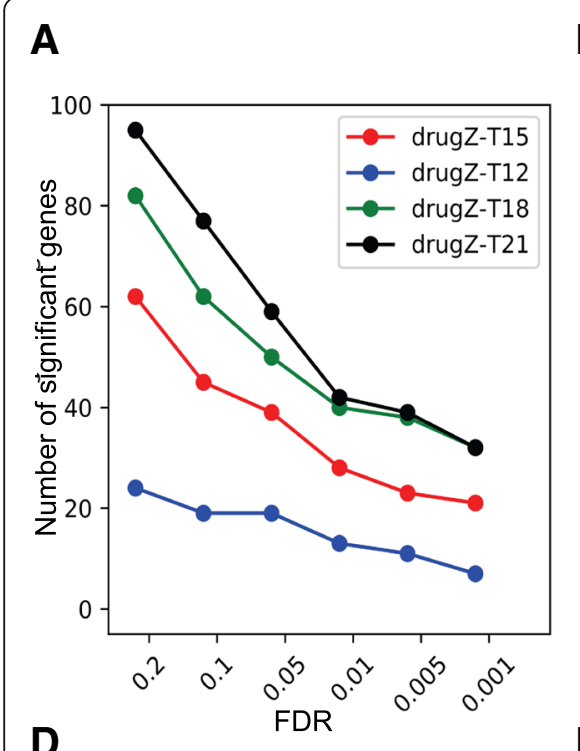

B

C
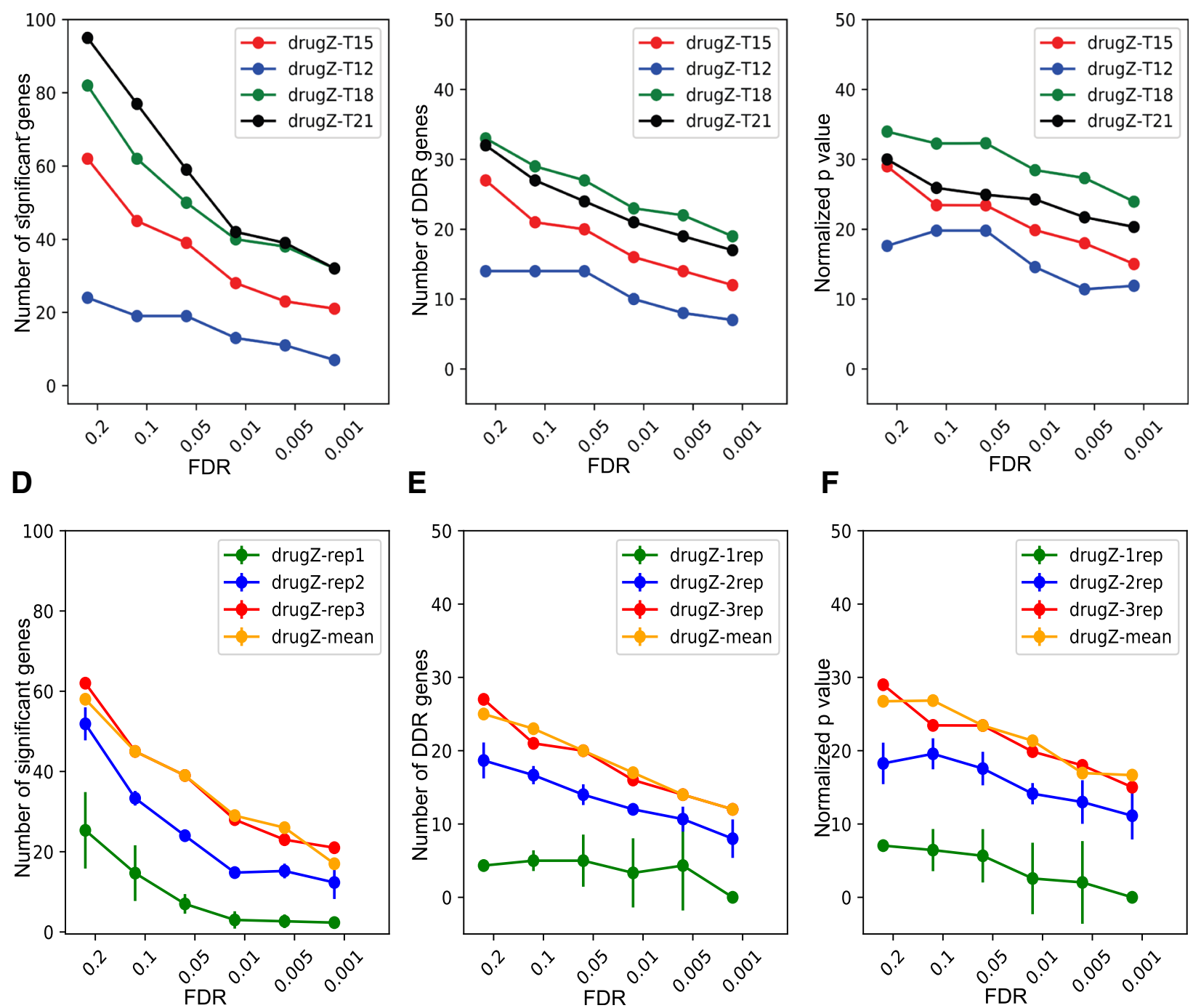

Fig. 2 Experimental design effects. a-c DrugZ performance across different time points for SUM149PT olaparib screen. a Number of raw hits. $\mathbf{b}$ Number of annotated DNA damage response (DDR) genes in hits. $\mathbf{c}-\log P$ values for DDR gene enrichment. $\mathbf{d}-\mathbf{f}$ DrugZ performance based on varying number of replicates. $\mathbf{d}$ Number of raw hits. e Number of annotated DNA damage response (DDR) genes in hits. $\mathbf{f}-\log P$ values for DDR gene enrichment. Rep1, 2, 3: all combinations of one, two, or three replicates, \pm s.d. Mean: comparing mean of drug-treated samples to the mean of control samples (unpaired approach)

synthetic lethality with the Fanconi anemia complex (Fig. 3f) and suppressor activity from the mismatch repair pathway (Fig. 3g, h). Together, these results recapitulate the biological drivers of temozolomide: mismatch repair is required for temozolomide cytotoxicity [42], while the Fanconi anemia pathway plays a major role in the repair of TMZ-induced damage [22, 43, 44] (Fig. 3i). We further conducted an independent screen of hTERT-immortalized RPE1 epithelial cells to determine genetic modifiers of the microtubule stabilizing agent vincristine. Drug transporter $A B C C 1$ (encoding multidrug resistance protein-1, or MRP1), a known marker for clinical resistance to vincristine $[45,46]$, is the top synthetic hit in our screen (Fig. 3j).

Finally, we reprocessed data from complementary CRISPRi/CRISPRa screens for modifiers of rigosertib activity [41] (Fig. 3k). As transcriptional activation and repression are expected to show opposite effects in a phenotypic screen, we plotted the drugZ results for the CRISPRi screen and the CRISPRa screen together (Fig. 3l). The microtubule stabilizing activity of TACC 3 and destabilizing activity of $K I F 2 C$, characterized extensively in [41], are both recovered by drugZ, along with tubulins TUBA1B and TUBB4 (Fig. 3l), consistent with rigosertib's activity as 


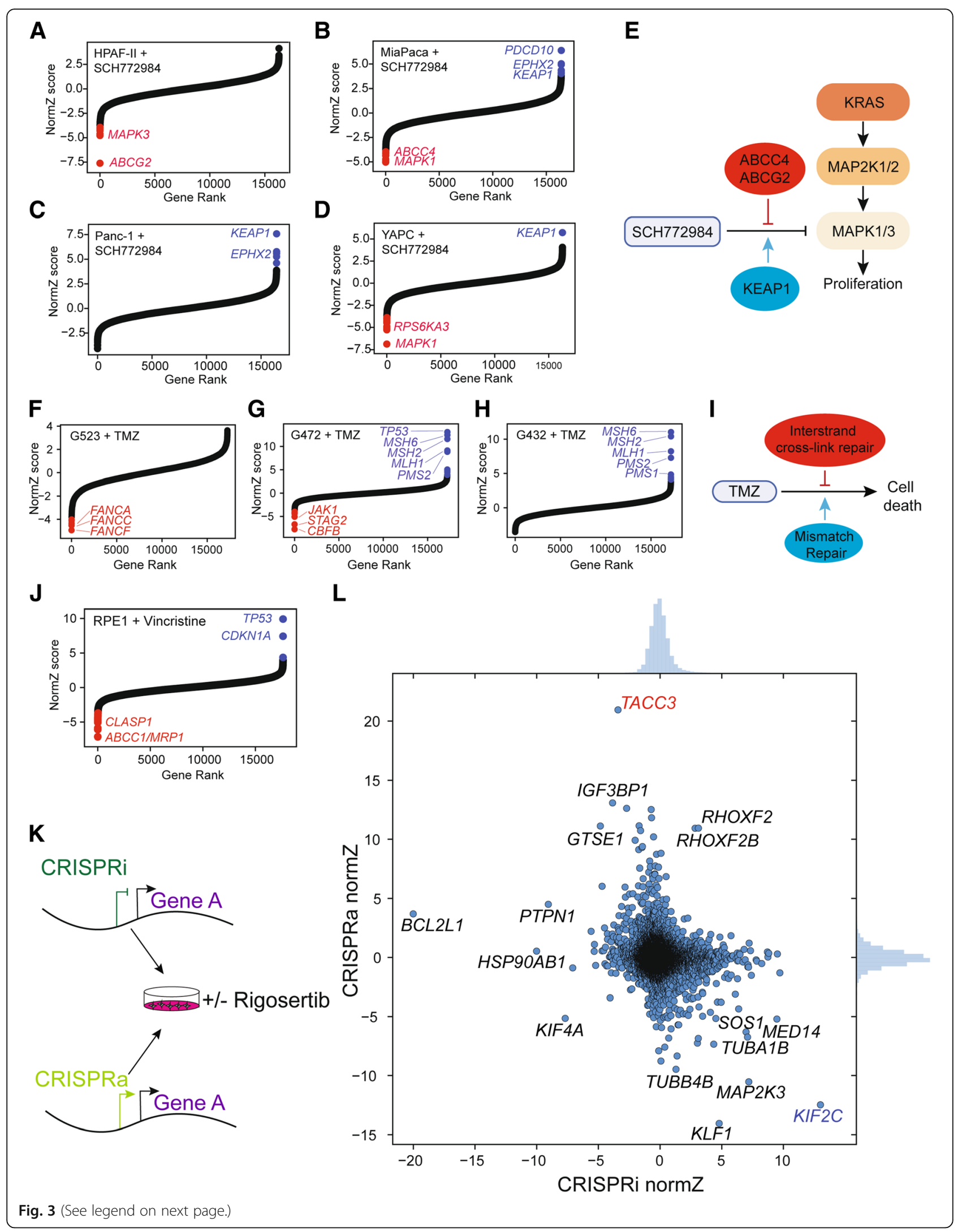


(See figure on previous page.)

Fig. 3 DrugZ effectiveness across diverse screens. a-d DrugZ-calculated normZ score is plotted vs. gene rank for SCH772984 screen in four KRAS pancreatic cancer cells cell lines. Synergistic/synthetic lethal (red) and suppressor/resistance (blue) interactions at FDR $<0.1$. e Network view of ERK inhibitor screens. Red, synthetic lethal interactions. Blue, suppressor interactions. $\mathbf{f}-\mathbf{h}$ Glioblastoma cell lines screened for chemogenetic interactions with temozolomide (TMZ), as described in [23]. i Pathway-level summary of modifiers of TMZ activity in glioblastoma cells. $\mathbf{j}$ hTERTRPE1 cells screened for modifiers of vincristine. $\mathbf{k}$ Experimental design of CRISPRi/CRISPRa screens for modifiers of rigosertib, as described in [41]. I DrugZ results of the combined rigosertib screens. Red/blue hits are characterized in [41]

a microtubule destabilizing agent. Importantly, these results confirm the applicability of drugZ beyond CRISPR knockout screens.

We noted that a small number of genes were unexpected repeat hits across several screens using different drug or small molecule perturbagens with disparate mechanisms of action. We screened hTERT-RPE1 cells with gemcitabine, a pyrimidine nucleoside analog, and analysis with drugZ reveals a synthetic lethal interaction with deoxythymidylate kinase DTYMK. DTYMK phosphorylates dTMP to dTDP, a key step in the synthesis-by-salvage pathway of dTTP [47] (Fig. 4a). However, suppressors of gemcitabine activity included NF2, TP53, AXIN1, and other known tumor suppressor genes (Fig. 4a) with no known role in nucleotide metabolism. This immortalized epithelial cell line carries wildtype alleles of these tumor suppressors, and their knockout in a CRISPR screen results in cell proliferation more rapid than wildtype cells. This is reflected in the essentiality profiles, as calculated by BAGEL [49]: essential genes have positive Bayes Factors, but tumor suppressors show extreme negative scores (Fig. 4b).

We hypothesized that such tumor suppressors might be systematic, nonspecific hits in drug-gene interaction screens. We re-analyzed other screens to understand this behavior across different cell backgrounds. The landmark CRISPR screen paper from Shalem et al. [5] includes a screen in BRAF-mutated A375 melanoma cells for resistance to vemurafenib and describes the discovery of NF2 as a novel suppressor of vemurafenib activity. DrugZ analysis confirms $N F 2$ as a strong hit in the screen, along with NF1 and several members of the mediator complex (Fig. 4c). Complementary analysis of the gene essentiality profile for A375 derived from Behan et al. [48] - the latest screens from the DepMap project are substantially superior to the first-generation screen performed in Shalem et al., as shown by precision-recall analysis (Additional file 1: Figure S5) shows that NF2 is the top ranked tumor suppressor in the screen, and furthermore, virtually every other
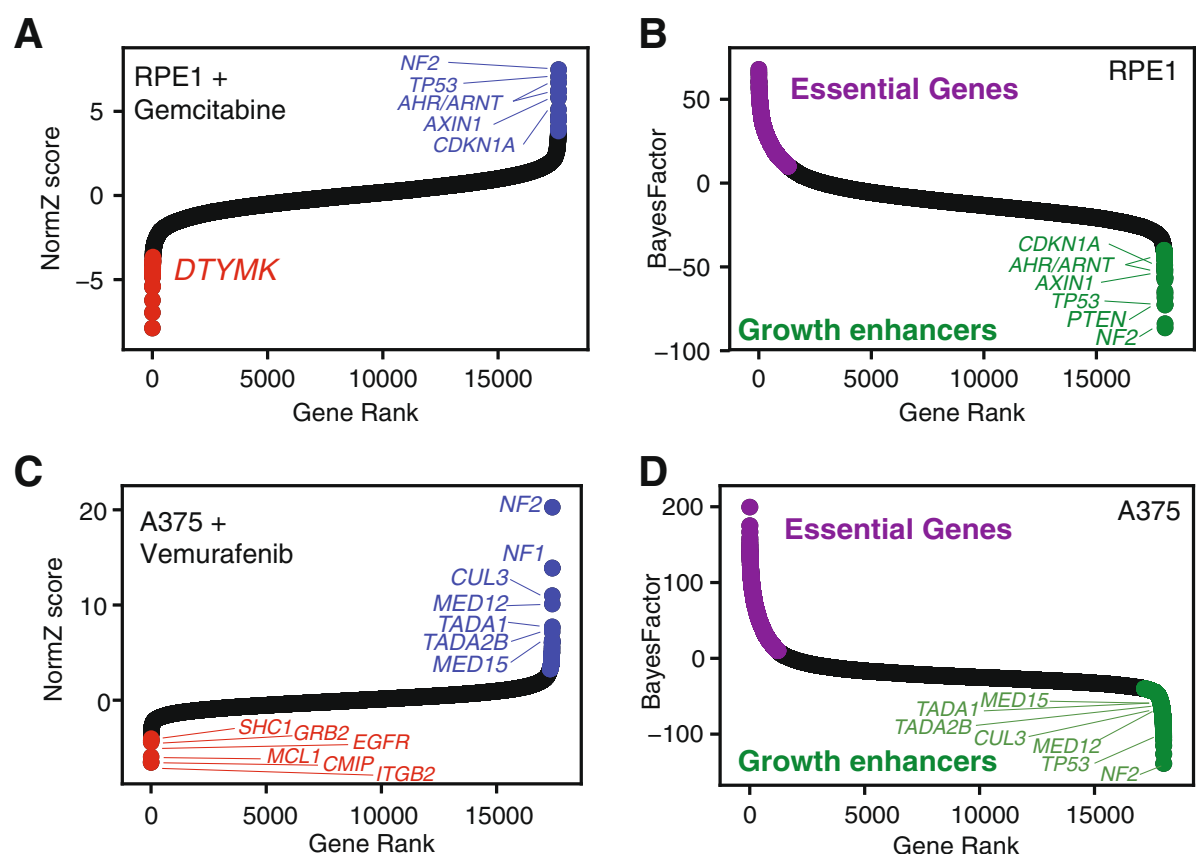

Fig. 4 Tumor suppressor genes are frequent drug suppressor hits. a normZ plot hTERT-RPE1 screen for modifiers of gemcitabine activity, colored as in Fig. 3. b Gene essentiality of untreated hTERT-RPE1 cells. Purple, essential genes. Green, genes whose knockout imparts a fitness advantage. c normZ plot of A375 melanoma cell line screen for vemurafenib modifiers; data from [5]. d Gene essentiality scores for A375; data from [48] 
vemurafenib suppressor hit shows enhanced cell fitness when knocked out (Fig. 4d). Interestingly, we detect $M C L 1$ and EGFR, as well as EGFR signal transduction components SHC1 and GRB2, as synthetic lethal with vemurafenib in this screen. Neither hit is reported in the original study, but both MCL1 [50] and EGFR [51, 52] have been characterized as routes of adaptive resistance to $B R A F$ inhibition in melanoma. These findings support the overall quality of the drug-gene interaction screen and our analysis of the data. We further note that TP53 and CDKN1A (p21) are the top suppressors in the RPE1 vincristine screen (Fig. 3j) and that TP53 is the top suppressor in the G472 temozolomide screen (Fig. 3g). G472 cells carry a wildtype p53 gene [23]. Collectively these results indicate that genes whose knockout imparts a growth advantage on cells are recurrent hits in drug-gene interaction screens, suggesting a drug-agnostic phenomenon rather than drug-specific resistance mechanisms.

\section{Conclusions}

Identifying the genetic drivers of drug effectiveness and resistance is critical to realize the promise of personalized medicine. Chemogenetic interaction screens in mammalian cells using CRISPR knockout libraries have so far been primarily used in a positive selection format to identify the genes, pathways, and mechanisms of acquired resistance to chemotherapeutic drugs. However, negative selection screens to identify the underlying architecture of drug-gene interactions have been difficult to carry out and to analyze in part due to the lack of robust analytical tools.

We describe the drugZ algorithm, which calculates a gene-level $Z$-score for pooled library CRISPR drug-gene interaction screens. By taking into account the moderate single mutant fitness defects associated with many genes involved in drug-gene interactions, the drugZ algorithm offers significantly improved sensitivity over contemporary analysis platforms. The algorithm was developed to exploit the additional resolving power we expected to gain from a paired-sample experimental design, but surprisingly this has virtually no effect on our results. We demonstrate the validity of our hits by showing the strong enrichment for genes involved in the DNA damage response in a screen for interactions with the PARP inhibitor olaparib and the precise detection of MAPK pathway effectors in an ERK inhibitor screen. We further show that both synergistic and suppressor interactions can be identified in the same screen, as the previously identified PARP resistance gene TP53BP1 and newly characterized SHLD1 (formerly C20orf196) are topranked suppressors of olaparib activity in BRCA1-mutant SUM149PT screens. Moreover, both synthetic targets MAPK1/3 and suppressor gene KEAP1 are identified in ERK inhibition screens. KEAP1 deletion or mutation is frequently found in KRAS-driven lung adenocarcinomas and may present an obstacle to ERK inhibitor therapy in these tumors.

Experimental design plays a critical role in the ability to accurately identify drug-gene interactions. Negative selection screens for synthetic lethal interactions require that cells be carried long enough for dropoutstypically growth defects rather than full synthetic lethals-to rise to statistical significance. Our results, concordant with known highly drug-specific differences in effect timing, suggest that there is value in collecting multiple time points to ensure that drug activity and genetic interaction are detectable and that traditional dose-response curves must be calculated over a time course relevant to the screen (e.g., at least two passages or several doublings).

Copy number amplifications have been widely shown to cause locus-specific, but not gene-specific, toxicity in CRISPR knockout experiments. This phenomenon can lead to false positives in screens for knockout fitness defects. However, drug-gene interaction screens measure whether, in the CRISPRko case, a double-strand break at a specific locus amplifies or suppresses the activity of a small molecule or other perturbagen. Amplification-specific artifacts should, in principle, show no difference between treated and control samples and should therefore not be a significant source of false positives. However, gRNA targeting amplified loci may rapidly drop out of a population of cells under library-induced selection; the absence of these loci at experimental end points (as measured by gRNA read counts) could feasibly mask the detection of drug-gene interactions, resulting in false negatives.

Despite these technical idiosyncrasies, chemogenetic interaction screens extend the utility of CRISPR genome-scale perturbation screens by enabling the systematic survey of the landscape of drug-gene interactions across cancer-relevant genetic backgrounds. Understanding this variation may lead to more precise therapies for patients as well as the development of synergistic drug combinations for genotype-specific treatments.

\section{Availability and requirements}

Project name: drugz

Project home page: https://github.com/hart-lab/drugz

Operating system: platform independent

Programming language: Python

Other requirements: Python v3.7 or higher; modules numpy, scipy, pandas.

License: MIT

No restriction for non-academic use 


\section{Additional file}

Additional file 1: Figure S1. DrugZ vs. other methods with olaparib screens in HeLa (A) and RPE1 (B) cells. Figure S2. DrugZ tunable parameters. Figure S3. DrugZ vs. DrugGS. Figure S4. Paired vs. non-paired approaches in three olaparib screens. Figure S5. Recall-precision plot of gene essentiality screens in A375 cells. (PDF $1842 \mathrm{~kb}$ )

\section{Acknowledgements}

The authors would like to thank Matej Usaj for practical assistance with software development and Edward Montes Benitez and Janice Whiting for their contributions to Hart Lab operations.

\section{Authors' contributions}

$M Z, K M, M M$, and LB performed drug-gene interaction screens under the supervision of JM, SA, DD, and TH. MC, GW, and WFL performed the initial development and testing of the drugZ algorithm. MC created the drugGS algorithm, refined drugZ, and wrote the final version of the software released here. MC and TH wrote the manuscript with editorial support from JM, SA, and especially DD. All authors approved the final version of the manuscript.

\section{Funding}

MC, GW, MM, LB, WFL, and TH were supported by MD Anderson Cancer Center Support Grant P30 CA016672 (the Bioinformatics Shared Resource) and the Cancer Prevention Research Institute of Texas (CPRIT) grant RR160032, and TH is supported by NIGMS grant R35GM130119. MZ is a Banting postdoctoral fellow. Work in the DD lab was funded through CIHR grant FDN143343, Canadian Cancer Society grant \#70389, as well as a Grantin-Aid from the Krembil Foundation. Work in JM lab is funded through CIHR grants 342551 and 365646. Work in SA lab was funded through a CIHR grant \#361837.

\section{Availability of data and materials}

All software described in this manuscript, as well as all data files used for analysis, are available (under the MIT license) at the Hart Lab github site and figshare:

https://github.com/hart-lab/drugz

https://github.com/hart-lab/druggs

https://figshare.com/projects/DrugZ_software_from_the_Hart_Lab/65582

\section{Ethics approval and consent to participate}

Not applicable.

\section{Consent for publication}

Not applicable.

\section{Competing interests}

T. Hart and D. Durocher are consultants for Repare Therapeutics. The remaining authors declare that they have no competing interests.

\section{Author details}

${ }^{1}$ Department of Bioinformatics and Computational Biology, The University of Texas MD Anderson Cancer Center, Houston, TX, USA. ${ }^{2}$ UTHealth Graduate School of Biomedical Sciences, The University of Texas MD Anderson Cancer Center, Houston, TX, USA. ${ }^{3}$ Lunenfeld-Tanenbaum Research Institute, Mount Sinai Hospital, 600 University Avenue, Toronto, ON M5G 1X5, Canada. ${ }^{4}$ Department of Pharmaceutical Sciences, Leslie Dan Faculty of Pharmacy, University of Toronto, Toronto, ON, Canada. ${ }^{5}$ Donnelly Centre, University of Toronto, Toronto, ON, Canada. ${ }^{6}$ Department of Molecular Genetics, University of Toronto, Toronto, ON M5S 3E1, Canada. ${ }^{7}$ Department of Biochemistry, University of Toronto, Toronto, ON, Canada.

Received: 19 February 2019 Accepted: 12 August 2019

Published online: 22 August 2019

\section{References}

1. Hartwell $\mathrm{LH}$. Integrating genetic approaches into the discovery of anticancer drugs. Science. 1997;278(5340):1064-8.
2. Giaever G, Chu AM, Ni L, Connelly C, Riles L, Véronneau S, et al. Functional profiling of the Saccharomyces cerevisiae genome. Nature. 2002;418(6896): 387-91.

3. Giaever G, Shoemaker DD, Jones TW, Liang H, Winzeler EA, Astromoff A, et al. Genomic profiling of drug sensitivities via induced haploinsufficiency. Nat Genet. 1999:21(3):278-83.

4. Jinek M, Chylinski K, Fonfara I, Hauer M, Doudna JA, Charpentier E. A programmable dual-RNA-guided DNA endonuclease in adaptive bacterial immunity. Science. 2012;337(6096):816-21.

5. Shalem O, Sanjana NE, Hartenian E, Shi X, Scott DA, Mikkelsen TS, et al. Genome-scale CRISPR-Cas9 knockout screening in human cells. Science. 2014;343(6166):84-7.

6. Wang T, Wei JJ, Sabatini DM, Lander ES. Genetic screens in human cells using the CRISPR-Cas9 system. Science. 2014;343(6166):80-4.

7. Doench JG, Fusi N, Sullender M, Hegde M, Vaimberg EW, Donovan KF, et al. Optimized sgRNA design to maximize activity and minimize off-target effects of CRISPR-Cas9. Nat Biotechnol. 2016;34(2):184-91.

8. Konermann S, Brigham MD, Trevino AE, Joung J, Abudayyeh OO, Barcena C, et al. Genome-scale transcriptional activation by an engineered CRISPR-Cas9 complex. Nature. 2015;517(7536):583-8.

9. Blondel CI, Park JS, Hubbard TP, Pacheco AR, Kuehl CI, Walsh MJ, et al. CRISPR/Cas9 screens reveal requirements for host cell sulfation and fucosylation in bacterial type III secretion system-mediated cytotoxicity. Cell Host Microbe. 2016;20(2):226-37.

10. Zhang R, Miner JJ, Gorman MJ, Rausch K, Ramage H, White JP, et al. A CRISPR screen defines a signal peptide processing pathway required by flaviviruses. Nature. 2016;535(7610):164-8.

11. Krall EB, Wang B, Munoz DM, Ilic N, Raghavan S, Niederst MJ, et al. KEAP1 loss modulates sensitivity to kinase targeted therapy in lung cancer. eLife. 2017:6:e18970.

12. le Sage C, Lawo S, Panicker P, Scales TME, Rahman SA, Little AS, et al. Dual direction CRISPR transcriptional regulation screening uncovers gene networks driving drug resistance. Sci Rep. 2017;7(1):17693.

13. Liao S, Davoli T, Leng Y, Li MZ, Xu Q, Elledge SJ. A genetic interaction analysis identifies cancer drivers that modify EGFR dependency. Genes Dev. 2017:31(2):184-96.

14. Zimmermann M, Murina O, Reijns MAM, Agathanggelou A, Challis R, Tarnauskaite Ž, et al. CRISPR screens identify genomic ribonucleotides as a source of PARP-trapping lesions. Nature. 2018;559(7713):285-9.

15. Wang C, Wang G, Feng X, Shepherd P, Zhang J, Tang M, et al. Genomewide CRISPR screens reveal synthetic lethality of RNASEH2 deficiency and ATR inhibition. Oncogene. 2018; [cited 2019 Feb 12]; Available from: http:// www.nature.com/articles/s41388-018-0606-4.

16. Estoppey D, Hewett JW, Guy CT, Harrington E, Thomas JR, Schirle M, et al. Identification of a novel NAMPT inhibitor by CRISPR/Cas9 chemogenomic profiling in mammalian cells. Sci Rep. 2017;7(1) [cited 2019 Mar 26]. Available from: http://www.nature.com/articles/srep42728.

17. Estoppey D, Lee CM, Janoschke M, Lee BH, Wan KF, Dong H, et al. The natural product cavinafungin selectively interferes with Zika and dengue virus replication by inhibition of the host signal peptidase. Cell Rep. 2017; 19(3):451-60.

18. Li W, Xu H, Xiao T, Cong L, Love Ml, Zhang F, et al. MAGeCK enables robust identification of essential genes from genome-scale CRISPR/Cas9 knockout screens. Genome Biol. 2014;15(12):554.

19. Robinson MD, McCarthy DJ, Smyth GK. edgeR: a Bioconductor package for differential expression analysis of digital gene expression data. Bioinformatics. 2010;26(1):139-40

20. Luo B, Cheung HW, Subramanian A, Sharifnia T, Okamoto M, Yang X, et al. Highly parallel identification of essential genes in cancer cells. Proc Natl Acad Sci. 2008:105(51):20380-5.

21. Colic M, Hart, Traver. Readcounts. figshare. Dataset. [Internet]. https://doi. org/10.6084/m9.figshare.8799215.v2. Available from: https://doi.org/10.6084/ m9.figshare.8799215.v2.

22. Yoshimoto K, Mizoguchi M, Hata N, Murata H, Hatae R, Amano T, et al. Complex DNA repair pathways as possible therapeutic targets to overcome temozolomide resistance in glioblastoma. Front Oncol. 2012;2 [cited 2019 Jul 7]. Available from: http://journal.frontiersin.org/article/10.3389/fonc.2012.00186/abstract

23. MacLeod G, Bozek DA, Rajakulendran N, Monteiro V, Ahmadi M, Steinhart Z et al. Genome-wide CRISPR-Cas9 screens expose genetic vulnerabilities and mechanisms of temozolomide sensitivity in glioblastoma stem cells. Cell Rep. 2019;27(3):971-986.e9. 
24. Hart T, Chandrashekhar M, Aregger M, Steinhart Z, Brown KR, MacLeod G, et al. High-resolution CRISPR screens reveal fitness genes and genotypespecific cancer liabilities. Cell. 2015;163(6):1515-26.

25. Bryant HE, Schultz N, Thomas HD, Parker KM, Flower D, Lopez E, et al. Specific killing of BRCA2-deficient tumours with inhibitors of poly (ADPribose) polymerase. Nature. 2005;434(7035):913-7.

26. Farmer H, McCabe N, Lord CJ, Tutt ANJ, Johnson DA, Richardson TB, et al. Targeting the DNA repair defect in BRCA mutant cells as a therapeutic strategy. Nature. 2005;434(7035):917-21.

27. Ashworth A. A synthetic lethal therapeutic approach: poly (ADP) ribose polymerase inhibitors for the treatment of cancers deficient in DNA doublestrand break repair. J Clin Oncol. 2008;26(22):3785-90.

28. Croft D, O'Kelly G, Wu G, Haw R, Gillespie M, Matthews L, et al. Reactome: a database of reactions, pathways and biological processes. Nucleic Acids Res. 2011;39(Database):D691-7.

29. Jaspers JE, Kersbergen A, Boon U, Sol W, van Deemter L, Zander SA, et al. Loss of 53BP1 causes PARP inhibitor resistance in Brcal -mutated mouse mammary tumors. Cancer Discov. 2013;3(1):68-81.

30. Dev H, Chiang T-WW, Lescale C, de Krijger I, Martin AG, Pilger D, et al. Shieldin complex promotes DNA end-joining and counters homologous recombination in BRCA1-null cells. Nat Cell Biol. 2018:20(8):954-65.

31. Ghezraoui H, Oliveira C, Becker JR, Bilham K, Moralli D, Anzilotti C, et al. 53BP1 cooperation with the REV7-shieldin complex underpins DNA structure-specific NHEJ. Nature. 2018;560(7716):122-7.

32. Mirman Z, Lottersberger F, Takai H, Kibe T, Gong Y, Takai K, et al. 53BP1RIF1-shieldin counteracts DSB resection through CST- and Pola-dependent fill-in. Nature. 2018;560(7716):112-6.

33. Noordermeer SM, Adam S, Setiaputra D, Barazas M, Pettitt SJ, Ling AK, et al. The shieldin complex mediates 53BP1-dependent DNA repair. Nature. 2018; 560(7716):117-21.

34. Aguirre AJ, Meyers RM, Weir BA, Vazquez F, Zhang C-Z, Ben-David U, et al. Genomic copy number dictates a gene-independent cell response to CRISPR/Cas9 targeting. Cancer Discov. 2016;6(8):914-29.

35. Koike-Yusa H, Li Y, Tan E-P, Velasco-Herrera MDC, Yusa K. Genome-wide recessive genetic screening in mammalian cells with a lentiviral CRISPRguide RNA library. Nat Biotechnol. 2014;32(3):267-73.

36. Tzelepis K, Koike-Yusa H, De Braekeleer E, Li Y, Metzakopian E, Dovey OM, et al. A CRISPR dropout screen identifies genetic vulnerabilities and therapeutic targets in acute myeloid leukemia. Cell Rep. 2016;17(4):1193-205.

37. Wang T, Yu H, Hughes NW, Liu B, Kendirli A, Klein K, et al. Gene essentiality profiling reveals gene networks and synthetic lethal interactions with oncogenic Ras. Cell. 2017;168(5):890-903 e15.

38. Hart T, Tong AHY, Chan K, Van Leeuwen J, Seetharaman A, Aregger M, et al. Evaluation and design of genome-wide CRISPR/SpCas9 knockout screens. G3. 2017;7(8):2719-27.

39. Ong SH, Li Y, Koike-Yusa H, Yusa K. Optimised metrics for CRISPR-KO screens with second-generation gRNA libraries. Sci Rep. 2017;7(1):7384.

40. Meyers RM, Bryan JG, McFarland JM, Weir BA, Sizemore AE, Xu H, et al. Computational correction of copy number effect improves specificity of CRISPRCas9 essentiality screens in cancer cells. Nat Genet. 2017;49(12):1779-84.

41. Jost M, Chen Y, Gilbert LA, Horlbeck MA, Krenning L, Menchon G, et al. Combined CRISPRi/a-based chemical genetic screens reveal that rigosertib is a microtubule-destabilizing agent. Mol Cell. 2017;68(1):210-23 e6.

42. Wang JYJ, Edelmann W. Mismatch repair proteins as sensors of alkylation DNA damage. Cancer Cell. 2006;9(6):417-8.

43. Kondo N, Takahashi A, Mori E, Noda T, Zdzienicka MZ, Thompson LH, et al. FANCD1/BRCA2 plays predominant role in the repair of DNA damage induced by ACNU or TMZ. PLoS One. 2011;6(5):e19659.

44. Chen CC, Taniguchi T, D'Andrea A. The Fanconi anemia (FA) pathway confers glioma resistance to DNA alkylating agents. J Mol Med. 2007;85(5):497-509.

45. Cole S, Bhardwaj G, Gerlach J, Mackie J, Grant C, Almquist K, et al. Overexpression of a transporter gene in a multidrug-resistant human lung cancer cell line. Science. 1992;258(5088):1650-4.

46. Godinot N, Iversen PW, Tabas L, Xia X, Williams DC, Dantzig AH, et al. Cloning and functional characterization of the multidrug resistanceassociated protein (MRP1/ABCC1) from the cynomolgus monkey. Mol Cancer Ther. 2003;2(3):307-16.

47. Arnér ESJ, Eriksson S. Mammalian deoxyribonucleoside kinases. Pharmacol Ther. 1995;67(2):155-86.
48. Behan FM, lorio F, Picco G, Gonçalves E, Beaver CM, Migliardi G, et al. Prioritization of cancer therapeutic targets using CRISPR-Cas9 screens. Nature. 2019;568(7753):511-6.

49. Hart T, Moffat J. BAGEL: a computational framework for identifying essential genes from pooled library screens. BMC Bioinformatics. 2016;17(1):164.

50. Fofaria NM, Frederick DT, Sullivan RJ, Flaherty KT, Srivastava SK. Overexpression of $\mathrm{MCl}-1$ confers resistance to $\mathrm{BRAF}^{\mathrm{V} 600 \mathrm{E}}$ inhibitors alone and in combination with MEK1/2 inhibitors in melanoma. Oncotarget. 2015 ;6(38). [cited 2019 Jul 7]. Available from: http://www.oncotarget.com/ fulltext/5755

51. Prahallad A, Sun C, Huang S, Di Nicolantonio F, Salazar R, Zecchin D, et al. Unresponsiveness of colon cancer to BRAF(V600E) inhibition through feedback activation of EGFR. Nature. 2012;483(7387):100-3.

52. Sun C, Wang L, Huang S, Heynen GJJE, Prahallad A, Robert C, et al. Reversible and adaptive resistance to BRAF(V600E) inhibition in melanoma. Nature. 2014;508(7494):118-22.

\section{Publisher's Note}

Springer Nature remains neutral with regard to jurisdictional claims in published maps and institutional affiliations.

\section{Ready to submit your research? Choose BMC and benefit from:}

- fast, convenient online submission

- thorough peer review by experienced researchers in your field

- rapid publication on acceptance

- support for research data, including large and complex data types

- gold Open Access which fosters wider collaboration and increased citations

- maximum visibility for your research: over $100 \mathrm{M}$ website views per year

At BMC, research is always in progress.

Learn more biomedcentral.com/submissions 